IMA PREPRint No. 1592 (1998)

\title{
Hyperbolic models for chemotaxis in 1-D
}

\author{
T. Hillen* $\quad$ A. Stevens ${ }^{\dagger}$
}

December 22, 1998

\begin{abstract}
:
In this paper we study a generalized Goldstein-Kac model in one space dimension for the chemotactic behavior of a biological species. This is motivated by the fact that the parabolic Keller-Segel model for chemotaxis allows arbitrarily large speeds of individual particles, whereas the hyperbolic model, which we consider here, deals with finite speeds. We will discuss the connections between the two models in detail, and focus especially on the relation between the crucial parameters - speed and turning rates in the hyperbolic case and diffusion rate and chemotactic sensitivity in the parabolic case. It turns out, that either the turning rates have to be different, or that the speed has to depend on the stimulus itself in order to observe chemotactic behavior. In case of constant speeds local and global existence for weak solutions of the hyperbolic model are shown. The proofs are based on a-priori estimates for the parabolic, resp. elliptic equation of the external stimulus. It turns out that boundedness of the gradient of the external signal is crucial to observe global existence. To compare the qualitative behavior of the hyperbolic model with the related parabolic one, we give some numerical examples.
\end{abstract}

Key words: chemotaxis, correlated random walks, hyperbolic equations, parabolic limit.

\section{Introduction}

Aggregation of individuals is a widespread phenomenon in biological systems. Reasons for this are, for instance, to search for food, to endure starvation conditions, to explore new regions, to reproduce or to give each other shelter. Each individual in the group senses its environment and/or the other members of its species and responds. Here we consider biological species which sense an external stimulus, produced by themselves, and move towards it. This behavior is called positive taxis. One distinguishes between chemotaxis, haptotaxis, aerotaxis, geotaxis and others, depending on what the type of

\footnotetext{
${ }^{*}$ University of Utah, Salt Lake City, UT, 84103 USA; e-mail: thomas.hillen@uni-tuebingen.de. Supported by the Deutsche Forschungsgemeinschaft.

${ }^{\dagger}$ This work was supported by the Deutsche Forschungsgemeinschaft during a stay of the second author at Stanford University, Stanford, CA 94305, USA. Present address: Institut für Angewandte Mathematik, Universität Heidelberg, INF 294, D-69120 Heidelberg, Germany; e-mail: stevens@iwr.uni-heidelberg.de.
} 
the external stimulus is.

In this paper we will consider chemotaxis, which describes the response of the individuals to an external chemical or its gradient. This phenomenon is experimentally well studied, e.g. for the slime mold amoebae of the species Dictyostelium discoideum (Dd) (compare e.g. Othmer and Schaap [38]) and for leukocytes (compare e.g. Gallin and Quie [14]). The slime mold amoebae aggregate under starvation conditions due to their excretion of cyclic-AMP. Finally they form so-called fruiting bodies, where they survive the unfavorable conditions as dormant spores. Soll [45] developed an experimental method to derive characteristic parameters for the motion of $\mathrm{Dd}$. These include velocity, directional changes, turns per unit time, angular velocity, and parameters for the shape of the amoebae. In Soll's studies it turned out, that the speed of amoebae and their turning behavior depends on both, the temporal and the spatial gradient of the cAMP concentration.

In this paper we present a hyperbolic model for chemotaxis in one space dimension, where the above mentioned parameters are introduced explicitly

$$
\begin{aligned}
u_{t}^{+}+\left(\gamma\left(s, s_{t}, s_{x}\right) u^{+}\right)_{x} & =-\mu^{+}\left(s, s_{t}, s_{x}\right) u^{+}+\mu^{-}\left(s, s_{t}, s_{x}\right) u^{-} \\
u_{t}^{-}-\left(\gamma\left(s, s_{t}, s_{x}\right) u^{-}\right)_{x} & =\mu^{+}\left(s, s_{t}, s_{x}\right) u^{+}-\mu^{-}\left(s, s_{t}, s_{x}\right) u^{-} \\
\tau s_{t} & =D s_{x x}+f\left(s, u^{+}+u^{-}\right), \tau \geq 0 .
\end{aligned}
$$

Here $u^{+}$denotes the density of the right moving part of the total population $u$ and $u^{-}$ denotes the density of the left moving part. The external stimulus is denoted by $s$. The turning rates $\mu^{+}$and $\mu^{-}$and the speed $\gamma$ depend on the chemoattractant $s$ and on its spatial and temporal gradient. We consider two kind of boundary conditions, either initial data with compact support on the whole line $\mathbb{R}$ or homogeneous Neumann boundary conditions on $[-1,1]$. The production and degradation of the external signal $s$ is given by the functional $f$.

Based on the Goldstein-Kac model ([15], [30]) for a one-dimensional correlated random walk, Segel [43] introduced a hyperbolic model of type (1) for chemotactic behavior. He studies increasing attractant concentration with constant speed $\gamma$, constant turning rate $\mu^{-}$, and $\mu^{+}$depending on the gradient of $s$ in characteristic direction. The external stimulus $s$ decays with a constant rate. His model has been used by Rivero e.a. [42] to describe experiments with flagellated bacteria and with leukocytes. The hyperbolic model proposed in (1) allows more general dependencies in the turning rates and the velocity, and especially comparison with the experiments of Soll ([45]). This comparison is not part of the present paper but will be done elsewhere.

Chen et al $[7,6]$ considered a model of the above type to describe experimental data for the movement of $E$. coli bacteria. In their model the bacterial speed is close to constant and the turning frequency depends on the temporal gradient of the external signal. Consequently bacteria "feel" spatial gradients by moving through them. The model is set into context with a one dimensional projection of the 3-D model of Alt [2], which we discuss later.

The connection of the hyperbolic model (1) to the well known parabolic chemotaxis equation of Keller and Segel (2) opens a wide field of interesting questions concerning 
scaling and modeling of crucial parameters. Some of them will be answered in section 2. For the special case of constant speed and for turning rates depending on $s$ and $s_{x}$ we prove local and global existence of solutions in $L^{\infty}$. To include dependence on $s_{t}$ a more detailed analysis is required. Here the preservation of total population size suffices to show existence of weak solutions in $L^{\infty}$. To control $s_{t}$ stronger preassumptions are required. If the speed depends on $s$ or its gradients we expect the formation of shocks, which will be treated elsewhere (see [27]).

Keller and Segel [31] introduced the following model for the dynamic behavior of a chemotactic species

$$
\begin{aligned}
u_{t}= & \nabla(A(u, s) \nabla u-\chi(u, s) u \nabla s) \\
\tau s_{t}= & \Delta s+f(u, s) \quad \text { for } x \in \mathbb{R}^{n}, t>0, \tau \geq 0 \\
& u(0, x)=u_{0}, \quad s(0, x)=s_{0} .
\end{aligned}
$$

The first equation describes the dynamics of the population density $u(t, x)$, the second equation diffusion, production and decay or annihilation of the external signal. If the chemotactic sensitivity $\chi(u, s)$ is positive, the population will move up gradients of the external signal. Their diffusion is enhanced or slowed down in dependence of the coefficient $A(u, s)$. The Keller-Segel model is based on the diffusion equation, hence arbitrary large particle speeds are involved. The hyperbolic model (1) explicitly includes finite propagation speed. Nevertheless in some situations parabolic equations describe spatial spread of the whole population quite well and the diffusion coefficient $A$ and the chemotactic sensitivity $\chi$ can be derived from experimental data (see e.g. Murray [35] for a collection of applications). On the other hand the parameters of the hyperbolic model - speed $\gamma$ and turning rates $\mu^{+}, \mu^{-}$- can be derived from the motion of individuals (tracking experiments). In this paper we show how the macroscopic and the microscopic parameters are related to each other. This has been done in the case of $E$. coli bacteria also by Chen et al [7].

For $A=$ const. the first rigorous derivation of the Keller-Segel system from an interacting stochastic many particle system has been done by Stevens [47]. The position of each particle fulfills a stochastic differential equation, where e.g. the chemotactic sensitivity of each particle depends on the other particles in a certain neighborhood around it. In the limit, when population size tends to infinity this range of interaction is rescaled in a moderate way.

A related approach to formally derive the Keller-Segel model, starts with a simplified version of a cellular automaton model for the gliding and aggregation of Myxobacteria, presented in Stevens [46], [48]. Like the slime mold amoebae these bacteria aggregate under starvation conditions, but the mechanism for this is still not known. In the automaton especially the slime trail following of the Myxobacteria is simulated, which is one of several possible explanations for their aggregation. The basic mechanism behind the automaton model for the slime trail following is related to a self-attracting reinforced random walk described by Davis [10]. He proved that a single particle localizes on the integers if an attractive reinforced non-diffusing substance is produced super linearly and does not, if the substance is produced only linearly (compare [10] for the 
exact conditions). The approximating parabolic chemotaxis-system nicely translates this localization behavior in a finite time blowup. The same behavior can be seen in a related hyperbolic model. This model will be our test problem in chapter 5, where we present some results of numerical simulations. Blowup for the classical Keller-Segel model has been analyzed in detail by several authors, e.g. Childress and Percus [9], Childress [8], Jäger and Luckhaus [29], Nagai [36], Herrero and Velazques [21], [20], Gajewski and Zacharias [13], Senba [44] and Levine and Sleeman [33], whose theoretical result was first shown numerically by Othmer and Stevens [39].

Equation (2) does not only describe aggregation of slime mold amoebae or bacteria, but is also used, for instance, to describe how cells in the immune system find their target. Moreover there are connections to astrophysics, compare e.g. Biler [4] and Biler and Nadzieja [5].

In absence of the nutrient $s$ and with constant speed $\gamma$ and constant turning rates $\mu^{ \pm}=\mu$, equation (1) is exactly the Goldstein-Kac model for a correlated random walk $([15,30])$, which under suitable conditions results in a damped wave equation (telegraph equation) for the total population density $u=u^{+}+u^{-}$

$$
\frac{1}{\mu} u_{t t}+u_{t}=\frac{\gamma^{2}}{\mu} u_{x x}
$$

For large speed and turning rate and constant quotient $B=\lim _{\gamma, \mu \rightarrow \infty} \gamma^{2} / \mu$ this equation formally approximates the diffusion equation $u_{t}=B u_{x x}$, (parabolic limit). When considering the Goldstein-Kac model combined with reaction and interaction of the populations, a semilinear reaction random walk system results

$$
\begin{aligned}
& u_{t}^{+}+\gamma u_{x}^{+}=\mu u^{-}-\mu u^{+}+f^{+}\left(u^{+}, u^{-}\right) \\
& u_{t}^{-}-\gamma u_{x}^{-}=\mu u^{+}-\mu u^{-}+f^{-}\left(u^{+}, u^{-}\right) .
\end{aligned}
$$

Reaction random walk systems are widely studied, e.g. by Holmes [28], Hadeler $[16,17,18,19]$ and Hillen $([22,23,24,25,26])$. The interplay between reaction and motion is discussed in detail in ([17]). Moreover boundary conditions on a finite interval are introduced; existence and uniqueness results for weak and classical solutions are given; energy methods are used to describe the asymptotic behavior of solutions; invariance and positivity results are given and traveling wave solutions are studied. Müller and Hillen [34] considered system (3) on $\mathbb{R}$. They rigorously proved the parabolic limit to a reaction diffusion equation for classical solutions and derived an initial layer estimate. Additionally a modulation equation is derived near a transcritical bifurcation and an approximation result is given.

Based on the linear model for a one dimensional correlated random walk, Pfistner [41] considered a model for the gliding behavior of Myxobacteria, where the turning rate depends on the bacterial density in a neighborhood of each individual. Then the equations loose their local structure and integro-differential equations follow.

This work is related to a paper by W. Alt [1]. He introduced a transport equation for 
chemotactic behavior in higher space dimensions. The external stimulus $s(t, x)$ is prescribed, so in difference to our approach, no explicit equation for $s$ is given. Considering experimental data of Brown and Berg ([3]), Alt estimated the distributions of the run time the turning angle in dependence on the external signal $s$ and on its gradients $s_{t}$ and $s_{x}$. Moreover he investigated the spectral properties of the turning operator and showed convergence to the classical chemotaxis equation (2) by using singular perturbation methods (parabolic limit). In the special case, where the turning rate is independent of the run time, a velocity-jump-chemotaxis process follows, which is also known as Stroocks equation (see [49] and [11] [12]). As shown by Ford and Cummings [11] [12], the Segel equation is a special case of Stroock's and Alt's equations.

Now we enlighten the connection between the present work and the model by Alt. If $\gamma=$ const. and for turning rates like we will consider in (16) the equations for $u^{ \pm}$in (1) are a special case of Alt's model. On the other hand Alt's model for one dimension fulfills the assumptions we will pose below - especially assumption (A4). Hence in this case our existence results cover Alt's equations. Besides taking a broader range of turning rates, we generalize the comparison between the hyperbolic models and the well known parabolic chemotaxis model to the case of variable speeds and consider diffusion, production and degradation of the external signal, which both has not been treated in [1].

In Othmer, Dunbar and Alt [37] general random motions are discussed: velocity jump processes and position jump processes. Alt's and Stroock's models are based on a velocity jump process.

The outline of this paper is as follows: In section 2 we discuss the hyperbolic chemotaxis model (1) in connection with the classical Keller-Segel model (2). If we choose appropriate parameter functions $\mu^{ \pm}$and $\gamma$ the Keller-Segel model is (at least formally) the parabolic limit of (1). Two different mechanisms can lead to aggregational behavior:

- the turning rates of the right and left moving population are different, due to non vanishing gradients of the external signal.

- the velocity $\gamma$ is depending on the external signal $s$.

In sections 3 and 4 we prove local and global existence results for constant speed $\gamma>0$. To focus on the influence of the dependencies of the turning rates $\mu^{ \pm}$and to avoid additional difficulties with nonlinear reproduction of $s$ we restrict ourselves to the case of linear reproduction $f\left(s, u^{+}+u^{-}\right)=\alpha\left(u^{+}+u^{-}\right)-\beta s$, with exception of section 5 , where numerical simulations for nonlinear reproduction dynamics are shown. Section 3 contains a-priori estimates which lead to a local existence theorem for weak solutions in $L^{\infty}$ for $\tau>0$. It turns out that for global existence an a-priori estimate for $s$ in $W^{1, \infty}$ is crucial, which makes the general case of nonlinear reproduction $f$ harder to analyze. If the turning rates are equally bounded, solutions exist globally, (Corollary 3.2). This excludes finite time blowup. The case of $\tau=0$ is considered separately in section 4 , where a global estimate is given for $\|s\|_{W^{1, \infty}}$, so solutions exist globally. In section 5 numerical examples are given, where globally existing patterns for linear reproduction $f$ and blow-up for nonlinear reproduction are shown and compared with the patterns of the related parabolic model, which has been considered in [39]. 


\section{The parabolic limit}

In this section we formally derive the parabolic chemotaxis-equations from the correlated random walk model given in (1). Here the velocity $\gamma$ and the turning rates $\mu^{ \pm}$depend on $s, s_{t}$ and $s_{x}$. So the dynamics of the total population density $u=u^{+}+u^{-}$and the flow $\gamma v$, where $v=u^{+}-u^{-}$are described by

$$
\begin{aligned}
& u_{t}+(\gamma v)_{x}=0, \\
& v_{t}+(\gamma u)_{x}=-\xi u-\eta v,
\end{aligned}
$$

where $\xi=\mu^{+}-\mu^{-}$and $\eta=\mu^{+}+\mu^{-}$. We differentiate the first equation with respect to $t$ and the second equation with respect to $x$. Since $(\gamma v)_{x t}=\gamma_{x} v_{t}+\gamma_{x t} v+\gamma_{t} v_{x}+\gamma v_{x t}$ and by using $v_{x t}=-(\gamma u)_{x x}-(\xi u)_{x}-(\eta v)_{x}$, we get a damped wave equation for $u$

$$
u_{t t}+\left(\eta-\frac{\gamma_{t}}{\gamma}\right) u_{t}-\left(\gamma(\gamma u)_{x}\right)_{x}-(\gamma \xi u)_{x}+\left(\gamma_{x t}-\frac{\gamma_{x} \gamma_{t}}{\gamma}-\gamma \eta_{x}\right) v=0
$$

If we assume

$$
\eta=\gamma_{t} / \gamma+c(t), \quad \text { such that } \quad \gamma=e^{\int\left(\mu^{+}+\mu^{-}-c(t)\right) d t}
$$

for a suitable function $c(t) \geq 0$, then $\gamma_{x t}-\frac{\gamma_{x} \gamma_{t}}{\gamma}-\gamma \eta_{x}=0$. To obtain the parabolic limit for high particle speeds we define

$$
\gamma_{\varepsilon}=\frac{1}{\varepsilon} \gamma \quad \text { and } \quad c_{\varepsilon}=\frac{1}{\varepsilon^{2}} c \quad \text { for } \quad 1 \geq \varepsilon>0
$$

When replacing $\gamma$ and $c$ in (5) by $\gamma_{\varepsilon}$ and $c_{\varepsilon}$ we get

$$
u_{t}=\left(\frac{\gamma}{c}(\gamma u)_{x}\right)_{x}+\left(\varepsilon \frac{\gamma}{c} \xi_{\varepsilon} u\right)_{x}+o(\varepsilon)
$$

which for $\varepsilon \rightarrow 0$ formally gives

$$
u_{t}=\left(A u_{x}-\chi u s_{x}\right)_{x}
$$

$$
\text { where } \quad A=\frac{\gamma^{2}}{c} \quad \text { and } \quad \chi s_{x}=-\frac{\gamma}{c}\left(\gamma_{x}+\lim _{\varepsilon \rightarrow 0} \varepsilon \xi_{\varepsilon}\right) .
$$

The growth of $\xi_{\varepsilon}$ in the limit $\varepsilon \rightarrow 0$ is crucial and will now be discussed in detail. We follow two lines of arguments. First we assume that $\mu^{ \pm}$and $\gamma$ are known from experimental data and derive expressions for the diffusion coefficient $A$ and the chemotactic sensitivity $\chi$ of the whole population, second we assume that $A$ and $\chi$ of the parabolic system are given and we choose turning rates $\mu_{\varepsilon}^{ \pm}$and speed $\gamma_{\varepsilon}$ such that the hyperbolic model $\left(1_{\varepsilon}\right)$ converges formally to the parabolic equation (2) for $\varepsilon \rightarrow 0$.

1. Assume that the speed $\gamma$ and the turning rates $\mu^{ \pm}$are prescribed. We define $\gamma_{\varepsilon}$ and $c_{\varepsilon}$ by (7). Then the corresponding sum of the turning rates $\eta_{\varepsilon}$ is given (6) with $\gamma_{\varepsilon}$ and $c_{\varepsilon}$ respectively. The parabolic limit is given by (9), (10). For $\chi$ to be finite with respect to the parabolic limit of $\varepsilon \rightarrow 0$ we assume that either $\xi_{\varepsilon}=\mu_{\varepsilon}^{+}-\mu_{\varepsilon}^{-}=O(1)$ or $O(1 / \varepsilon)$. In the following we consider different situations which are biologically relevant. 
(a) If $\gamma=\gamma(s) \geq 0$ and if we assume, that $\xi_{\varepsilon}=\mu_{\varepsilon}^{+}-\mu_{\varepsilon}^{-}=O(1)$ then

$$
\chi s_{x}=-\frac{\gamma}{c} \dot{\gamma} s_{x}
$$

and the limiting parabolic equation reads

$$
u_{t}=\left(\frac{\gamma}{c}\left(\gamma u_{x}+\dot{\gamma} s_{x} u\right)\right)_{x} .
$$

If $\dot{\gamma}(s)<0$, which means that the population slows down, when the concentration of $s$ is higher. This results in an aggregative effect. A net flow of the population in direction of higher concentrations of $s$ follows, which is called (positive taxis). Note that the case $\mu_{\varepsilon}^{+}=\mu_{\varepsilon}^{-}$is included here.

If $\dot{\gamma}(s)>0$, which means that higher concentrations of $s$ enhance the speed then the population has a higher tendency to spread out than when only diffusing. This behavior is called negative taxis. Hence the population glides in direction of smaller spatial gradients of $s$.

(b) Assume $\gamma=$ const. and

$$
\xi_{\varepsilon}=\frac{1}{\varepsilon^{2}} \sum_{k=1}^{\infty} \varphi_{k}\left(\varepsilon s_{x}\right)^{k},
$$

where the coefficients $\varphi_{k}$ may depend on $s$ and $s_{t}$, then

$$
\chi s_{x}=-\lim _{\varepsilon \rightarrow 0} \varepsilon \xi_{\varepsilon} .
$$

Hence the chemotactic sensitivity $\chi=-\varphi_{1}$ is the negative first order approximation of the difference of the turning rates in $s_{x}$. So we get

$$
u_{t}=\left(\frac{\gamma^{2}}{c} u_{x}+\varphi_{1} s_{x} u\right)_{x}
$$

Here the swarming or aggregative behavior depends exactly on the sign of the difference between the turning frequencies.

If $s_{x}>0$ and $\varphi_{1}<0$, then the right moving population, which is moving up the gradient, turns less often than the left moving one, which is moving down the gradient. This is the basic behavior also observed during experiments for chemotactic behavior.

If $s_{x}>0$ and $\varphi_{1}>0$, then the right moving population turns more often than the left moving one, which enhances the spreading out of the total population. The above expansion of $\xi_{\varepsilon}$ is important, when considering turning rates depending nonlinearly on the gradient of $s$. Especially saturation effects can be taken into account.

2. Now let $A>0$ and $\chi$ be given. For $0<\varepsilon \leq 1$ we choose $\gamma_{\varepsilon}\left(s, s_{t}, s_{x}\right)$ such that

$$
\lim _{\varepsilon \rightarrow 0} \varepsilon \gamma_{\varepsilon}=\sqrt{A}
$$


and define

$$
\mu_{\varepsilon}^{ \pm}:=\frac{1}{2}\left(\frac{\gamma_{\varepsilon t}}{\gamma_{\varepsilon}}+\left(\frac{1}{\varepsilon^{2}}\right) \mp \frac{1}{\varepsilon}\left(\frac{\chi_{x}}{\sqrt{A}}-(\sqrt{A})_{x}\right)\right)^{+}
$$

If $\gamma$ depends on $s$ then the turning rates depend not only on $s$ and $s_{x}$ but also on $s_{t}$. The exponent "+" indicates the positive part of this expression. This is important for the hyperbolic model to have positive turning rates. On the other hand it does not matter for the parabolic limit, since then $1 /\left(2 \varepsilon^{2}\right)$ dominates the other terms.

For constant Diffusion rate $A$ we choose $\gamma_{\varepsilon}:=\frac{1}{\varepsilon} \sqrt{A}$ and define

$$
\mu_{\varepsilon}^{ \pm}:=\frac{1}{2 \varepsilon}\left(\frac{1}{\varepsilon} \mp \frac{\chi s_{x}}{\sqrt{A}}\right)^{+} \text {. }
$$

\section{Assumptions and Results}

In this section we give the detailed assumptions and the existence results which are proved in the following sections. We consider the one dimensional hyperbolic model (1) with constant speed $\gamma$, linear production of $s$ and turning rates independent of $s_{t}$.

$$
\begin{aligned}
u_{t}^{+}+\gamma u_{x}^{+} & =-\mu^{+}\left(s, s_{x}\right) u^{+}+\mu^{-}\left(s, s_{x}\right) u^{-}, \\
u_{t}^{-}-\gamma u_{x}^{-} & =\mu^{+}\left(s, s_{x}\right) u^{+}-\mu^{-}\left(s, s_{x}\right) u^{-}, \\
\tau s_{t} & =D s_{x x}-\beta s+\alpha\left(u^{+}+u^{-}\right) .
\end{aligned}
$$

For $\tau \neq 0$ we assume $\tau=1$ without loss of generality. To include the time derivative of $s$ a more detailed regularity analysis of the whole system is required. Here we start with the control of the $L^{1}$ norm (conservation of total population size) to show $L^{2}$ estimates for $u^{ \pm}$and $W^{1, \infty}$ estimates for $s$. This finally leads to local existence in $L^{\infty}$.

We consider two kinds of boundary conditions.

(bc1) $\Omega=\mathbb{R}$ and $u_{0}, v_{0}, s_{0}$ have compact support.

(bc2) $\Omega=[-1,1]$ and homogeneous Neumann boundary conditions:

$$
u^{+}(-1)=u^{-}(-1), \quad u^{-}(1)=u^{+}(1), \quad s_{x}(-1)=s_{x}(1)=0 .
$$

We assume:

(A1) Nonnegative initial values:

$$
\begin{array}{r}
u_{0}^{+}, u_{0}^{-} \in L^{2}(\Omega) \cap L^{\infty}(\Omega), s_{0} \in W^{1,2}(\Omega) \cap W^{1, \infty}(\Omega) \text { and } \\
u_{0}^{+} \geq 0, \quad u_{0}^{-} \geq 0, \quad s_{0} \geq 0 .
\end{array}
$$

(A2) Nonnegative turning rates: $\mu^{ \pm} \geq 0$.

(A3) Symmetry of the turning rates, with respect to $s_{x}: \mu^{+}\left(s, s_{x}\right)=\mu^{-}\left(s,-s_{x}\right)$.

(A4) Local Lipschitz continuity and boundedness of $\mu^{ \pm}: L^{\infty}(\Omega) \times L^{\infty}(\Omega) \rightarrow \mathbb{R}$ :

$$
0 \leq \mu^{ \pm}\left(s, s_{x}\right) \leq m\left(1+\|s\|_{W^{1, \infty}}\right), \text { for } m>0 .
$$


Assumption (A4) covers the special turning rates given in (16). Moreover the parameter chosen by W.Alt [2] fit into this condition.

Theorem 3.1 Assume (A1)-(A4) for system (17), with boundary conditions (bc1) or (bc2). Then there exists a unique solution

$$
\left(u^{+}, u^{-}, s\right) \in L^{\infty}\left(\left[0, T_{\max }\right), L^{\infty}(\Omega)^{2} \times W^{1, \infty}(\Omega)\right)
$$

for a maximal $T_{\max }>0$. If $T_{\max }<\infty$ then

$$
\lim _{t \rightarrow T_{\max }}\|s(t, .)\|_{W^{1, \infty}}=\infty .
$$

The proof of Theorem 3.1 is given in section 4.2. This theorem is a first existence result for a hyperbolic chemotaxis equation, where the external signal $s$ is a dynamical variable. It shows that the gradient of the external signal triggers possible blow-up's. Existence results for the parabolic chemotaxis equation are well known. The latest one is that by Herrero and Velázques [20], who considered rotationally symmetric solutions in a 2-D model, which blow up in finite time. Since in (17) the parabolic equation of the external signal is coupled to a hyperbolic system the existence theory becomes interesting. The hyperbolic system shows no regularity properties but due to the assumptions, e.g. (A4) one can expect that an a-priori bound on $\|s\|_{W^{1, \infty}}$ will suffice to prove existence of solutions. To derive this estimate from an $L^{1}$ estimate for the solutions, we use parabolic regularization of the equation for $s$ and a bootstrapping argument (Theorem 4.5). Then we prove local existence by a contraction mapping principle. The second statement in Theorem 3.1 gives a criterion which may be used to exclude finite time blow up. Especially for uniformly bounded turning rates this leads to global existence. Here we replace (A4) by

(A4') Local Lipschitz continuity of $\mu^{ \pm}: L^{\infty}(\Omega) \times L^{\infty}(\Omega) \rightarrow \mathbb{R}$, and uniform boundedness:

$$
0 \leq \mu^{ \pm}\left(s, s_{x}\right) \leq m^{*}, \quad \text { for all } s \in W^{1, \infty} .
$$

In experiments for the motion of $\mathrm{Dd}$, Soll [45] indeed could give explicit upper bounds for the turning rates.

Corollary 3.2 Assume (A1)-(A3) and (A4') for system (17), with boundary conditions (bc1) or (bc2). Then there exists a unique solution

$$
\left(u^{+}, u^{-}, s\right) \in L^{\infty}\left([0, \infty), L^{\infty}(\Omega)^{2} \times W^{1, \infty}(\Omega)\right) .
$$

The proof of this corollary is given in section 4.3. Of course this case excludes comparison to the parabolic limit, since the turning rates are uniformly bounded. 
The situation $\tau=0$ will be considered separately. Here system (17) reads

$$
\begin{aligned}
u_{t}^{+}+\gamma u_{x}^{+} & =-\mu^{+}\left(s, s_{x}\right) u^{+}+\mu^{-}\left(s, s_{x}\right) u^{-}, \\
u_{t}^{-}-\gamma u_{x}^{-} & =\mu^{+}\left(s, s_{x}\right) u^{+}-\mu^{-}\left(s, s_{x}\right) u^{-}, \\
D s_{x x} & =\beta s-\alpha\left(u^{+}+u^{-}\right) .
\end{aligned}
$$

Without loss of generality we can assume $D=1$. Again we study both kinds of boundary conditions, (bc1) and (bc2). We assume (A1)-(A4), without prescribing initial data for $s$. This model refers to the observation that the diffusion of $s$ is fast compared to the motion of the particles. Hence we assume that at each time the external signal $s$ is in a quasi stationary state. Under this assumption the parabolic equations for chemotaxis in $n$ dimensions were considered by several authors, see e.g. Jäger, Luckhaus [29], Nagai [36] or Herrero, Velazquez [21]. If $\tau=0$ the external signal $s$ is given as a solution of an elliptic equation and we will get a global $L^{\infty}$ estimate for the gradient $s_{x}$. This acts as a key estimate for global existence.

Theorem 3.3 Assume (A1)-(A4) for system (18) without prescribing initial conditions for $s$. Consider the boundary conditions ( $\mathrm{bc} 1)$ or ( $\mathrm{bc} 2$ ). Then there exists a unique solution

$$
\left(u^{+}, u^{-}, s\right) \in L^{\infty}\left([0, \infty), L^{\infty}(\Omega)^{2} \times W^{1, \infty}(\Omega)\right) .
$$

The proof mimics the one of Theorem 3.1, where the parabolic a-priori estimates of Theorem 4.5 are replaced by elliptic ones, which are proven in Lemma 5.3 . The final proof of Theorem 3.3 is given in section 5.2.

\section{The case of $\tau \neq 0$}

\subsection{A-priori estimates}

Since for the hyperbolic system (17) the total population density is preserved, the $L^{1}$-norm is a natural measure for solutions. However to estimate the solution $s(t, x)$ of the parabolic equation $\tau s_{t}=D s_{x x}-\beta s+\alpha\left(u^{+}+u^{-}\right)$in $W^{1, \infty}$ it is not sufficient to assume that $u^{+}(t,),. u^{-}(t,.) \in L^{1}(\Omega)$. Here we prove local a-priori estimates for $u^{+}, u^{-}$ in $L^{2}$ and for $s$ in $W^{1, \infty}$ simultaneously using a bootstrapping argument. For $\tau=0$ in (17) an $L^{\infty}$ estimate for $s_{x}$ follows easily (compare section 5 or e.g. Nagai [36]). Here $s$ satisfies a parabolic equation and hence $\left\|s_{x}\right\|_{\infty}$ is bounded by the $L^{2}$-norm of the solution $u^{ \pm}$(see Lemma 4.1), which we use to estimate $s_{x}$ at least for small times.

Assume that solutions of (17) with the prescribed boundary conditions exist in the usual weak sense in $L^{1}\left(\left[0, t_{0}\right) \times \Omega\right)$ for some finite time $t_{0}>0$. First we consider the parabolic equation for the external signal $s$ separately. The solution $s$ of the initial value problem

$$
\begin{aligned}
s_{t} & =D s_{x x}-\beta s, \\
s(0, .) & =s_{0} \in W^{1,2}(\Omega) \cap W^{1, \infty}(\Omega),
\end{aligned}
$$


is given by $s(t, x)=T(t) s_{0}(x)=e^{-\beta t} T_{0}(t) s_{0}(x)$ for $0 \leq t<t_{0}$. Here $T_{0}(t)$ is the solution semigroup of the heat equation with Neumann boundary conditions in case of (bc2) and on $L^{2}(\mathbb{R})$ in case of $(\mathrm{bc} 1)$. Then the solution of the initial boundary value problem

$$
\begin{aligned}
s_{t} & =D s_{x x}-\beta s+\alpha\left(u^{+}+u^{-}\right), \\
s(0, .) & =s_{0} \in W^{1,2}(\Omega) \cap W^{1, \infty}(\Omega)
\end{aligned}
$$

is given by

$$
s(t, x)=T(t) s_{0}(x)+\alpha \int_{0}^{t} T(t-\theta)\left(u^{+}+u^{-}\right)(\theta, x) d \theta, \quad \text { for } 0 \leq t<t_{0} .
$$

It is well known (see, e.g. Taylor, p. 274 [50]) that for both types of boundary conditions $T(t): L^{1}(\Omega) \rightarrow L^{1}(\Omega)$ and $T(t): L^{\infty}(\Omega) \rightarrow L^{\infty}(\Omega)$ are continuous and their operator norm is bounded by a time independent constant $C>0$. Moreover

$$
\begin{array}{llll}
T(t): & L^{1}(\Omega) \rightarrow W^{1, \infty}(\Omega) & \text { has norm } & C t^{-1}, \\
T(t): & L^{2}(\Omega) \rightarrow W^{1, \infty}(\Omega) & \text { has norm } & C t^{-3 / 4}, \\
T(t): & L^{\infty}(\Omega) \rightarrow W^{1, \infty}(\Omega) & \text { has norm } & C t^{-1 / 2},
\end{array}
$$

where the constants $C>0$ may be different.

Lemma 4.1 Assume that for $0 \leq t<t_{0}$ the functions $u^{+}(t,),. u^{-}(t,.) \in L^{2}(\Omega) \cap L^{\infty}(\Omega)$. Then

$$
\begin{aligned}
\|s(t, .)\|_{W^{1, \infty}} & \leq C_{2}\left(\left\|s_{0}\right\|_{W^{1, \infty}}+t^{1 / 4}\left\|u^{+}(t, .)+u^{-}(t, .)\right\|_{2}\right) \\
\|s(t, .)\|_{W^{1, \infty}} & \leq C_{\infty}\left(\left\|s_{0}\right\|_{W^{1, \infty}}+t^{1 / 2}\left\|u^{+}(t, .)+u^{-}(t, .)\right\|_{\infty}\right)
\end{aligned}
$$

with some constants $C_{2}, C_{\infty}>0$.

If $u^{ \pm}(t, x) \geq 0$ for all $(t, x) \in\left[0, t_{0}\right) \times \Omega$ then $s(t, x) \geq 0$.

Proof: The estimates follow from the properties of the semigroup $T(t)$ and the representation (19). Since $T(t)$ is a positive semigroup the second statement is obvious.

Positivity of $u^{+}$and $u^{-}$follows from the assumptions (A1)-(A3):

Lemma 4.2 Assume (A1)-(A3) then the solution satisfies $u^{ \pm}(t, x) \geq 0$ as long as it exist.

Proof: First we consider solutions in $u^{+}, u^{-} \in C^{1}\left(\left[0, t_{0}\right) \times \Omega\right)$. For this we separate the two boundary conditions.

1. For Neumann boundary conditions (bc2) the statement follows directly from an invariance principle for hyperbolic random walk systems (see [26]).

2. For initial data with compact support, (bc1), the hyperbolic equations in (17) preserve this property for $\left(u^{+}, u^{-}\right)(t,$.$) . (This is not true for s$ ). So for given $T \in\left(0, T_{\max }\right)$ there exists a ball with radius $R(T)$ which contains the support of $\left(u^{+}, u^{-}\right)(t,$.$) for each 0 \leq t \leq T$. Hence the solutions satisfy

$$
u^{+}(t,-R(T))=0, \quad u^{-}(t, R(T))=0 .
$$

Again the result in [22] applies. 
To observe this result for $L^{1}$-solutions, we approximate with $C^{1}$-solutions using semigroup properties of the hyperbolic equation for $u^{ \pm}$. Let $\left(u^{+}, u^{-}, s\right)$ denote the (weak) solution of (17), which we assume to exist. Now fix $s(t, x) \in W^{1, \infty}$ and consider the initial value problem

$$
\begin{aligned}
v_{t}^{+}+\gamma v_{x}^{+} & =-\mu^{+}\left(s, s_{x}\right) v^{+}+\mu^{-}\left(s, s_{x}\right) v^{-} \\
v_{t}^{-}-\gamma v_{x}^{-} & =\mu^{+}\left(s, s_{x}\right) v^{+}-\mu^{-}\left(s, s_{x}\right) v^{-} \\
v^{ \pm}(0, x) & =v_{0}^{ \pm}(x)
\end{aligned}
$$

In [22] it is shown, that the linear part of equation (22) defines a strongly continuous semigroup on $L^{p}(\Omega)$ for each $1 \leq p<\infty$. By a standard perturbation argument (see e.g. Pazy [40]) this remains true for (22) since $\mu^{ \pm}$is bounded (see (A4)). Let $Q(t)$ denote the solution semigroup of (22). For $n \in \mathbb{N}$ we choose initial data $\left(u_{0, n}^{+}, u_{0, n}^{-}\right) \in C^{1}(\Omega)$ which approximate $\left(u_{0}^{+}, u_{0}^{-}\right)$in $L^{1}(\Omega)$. In case of Neumann boundary conditions we assume that in addition the compatibility conditions

$$
\left(u_{0, n}^{+}(-1)+u_{0, n}^{-}(-1)\right)_{x}=\left(u_{0, n}^{+}(1)+u_{0, n}^{-}(1)\right)_{x}=0
$$

are satisfied. Let $\left(u_{n}^{+}(t, x), u_{n}^{-}(t, x)\right):=Q(t)\left(u_{0, n}^{+}(x), u_{0, n}^{-}(x)\right)$ denote the corresponding solutions. These solutions are classical and from [26] it follows that $u_{n}^{ \pm} \geq 0$ for all $n \in \mathbb{N}$. Since the semigroup $Q(t)$ is continuous the limit $u^{ \pm}=\lim _{n \rightarrow \infty} u_{n}^{ \pm}$also satisfies $u^{ \pm} \geq 0$.

Corollary 4.3 Given the conditions of Lemma 4.2 we have

$$
u(t, x) \geq|v(t, x)|
$$

for all $0 \leq t<T_{\max }$ and $x \in \Omega$.

Now we estimate the $L^{2}$-norm of $u^{ \pm}$in terms of the $W^{1, \infty}{ }_{-}$norm of $s$ :

Lemma 4.4 Assume (A1)-(A4) and assume that $\left(u^{+}, u^{-}, s\right) \in C^{1}\left(\left[0, t_{0}\right), L^{\infty}(\Omega)^{3}\right)$ is a solution of $(17)$ with $s(t,.) \in W^{1, \infty}(\Omega)$. Then

$$
\frac{d}{d t}\left\|\left(u^{+}, u^{-}\right)(t, .)\right\|_{2}^{2} \leq 2 m\left(1+\|s(t, .)\|_{W^{1, \infty}}\right)\left\|\left(u^{+}, u^{-}\right)(t, .)\right\|_{2}^{2} .
$$

Proof: We use the $(u, v)$-notation (4):

$$
\begin{aligned}
\frac{d}{d t}\|(u, v)(t, .)\|_{2}^{2} & =2 \int_{\Omega}\left(u u_{t}+v v_{t}\right) d x \\
& =2 \int_{\Omega}\left(-\gamma u v_{x}-\gamma v u_{x}-\xi u v-\eta v^{2}\right) d x
\end{aligned}
$$

For (bc1) as well as (bc2) we have

$$
\gamma \int_{\Omega}\left(u v_{x}+v u_{x}\right) d x=0
$$


With Corollary 4.3 it follows that

$$
\left|\int_{\Omega} \xi u v d x\right| \leq\left|\int_{\Omega} \xi u^{2} d x\right| \leq\|\xi(t, .)\|_{\infty}\|u(t, .)\|_{2}^{2}
$$

Using assumption (A4) we estimate $\xi=\mu^{+}-\mu^{-}$as

$$
\|\xi(t, .)\|_{\infty} \leq m\left(1+\|s(t, .)\|_{W^{1, \infty}}\right) .
$$

Since $\eta=\mu^{+}+\mu^{-}>0$ it follows from (24) that

$$
\frac{d}{d t}\|(u, v)(t, .)\|_{2}^{2} \leq 2 m\left(1+\|s(t, .)\|_{W^{1, \infty}}\right)\|(u, v)(t, .)\|_{2}^{2}
$$

which gives $(23)$ with $u^{+}=1 / 2(u+v)$ and $u^{-}=1 / 2(u-v)$.

In Lemma 4.1, (20) we showed an $L^{\infty}$-estimate of $s_{x}$ depending on the $L^{2}$-norm of $u^{ \pm}$, whereas here we estimated the $L^{2}$-norm in terms of the $L^{\infty}$-norm of $s_{x}$. We use a bootstrapping argument to show a-priori estimates for both norms:

Theorem 4.5 Assume that $\left(u^{+}, u^{-}, s\right) \in L^{1}\left(\left[0, t_{0}\right) \times \Omega\right)^{3}$ is a (weak) solution of (17) with boundary conditions (bc1) or (bc2) and suppose that (A1)-(A4) are satisfied. Then for each constant $C^{*} \geq\left\|\left(u_{0}^{+}, u_{0}^{-}\right)\right\|_{2}^{2}$ there exists a time $t^{*}>0$ with $t^{*}=$ $t^{*}\left(C^{*},\left\|\left(u_{0}^{+}, u_{0}^{-}\right)\right\|_{2},\left\|s_{0}\right\|_{W^{1, \infty}}\right)$, where $t^{*}$ increases if $C^{*}$ increases, such that

$$
\left\|\left(u^{+}, u^{-}\right)(t, .)\right\|_{2}^{2} \leq C^{*} \quad \text { and } \quad\|s(t, .)\|_{W^{1, \infty}} \leq C^{* *}
$$

for all $0 \leq t<\min \left\{t_{0}, t^{*}\right\}$, and

$$
C^{* *}=C\left(1+\left\|s_{0}\right\|_{W^{1, \infty}}+\left(t^{*}\right)^{1 / 4} C^{*}\right) .
$$

Proof: First assume that $\left(u^{+}, u^{-}\right) \in C^{1}\left(\left[0, t_{0}\right), L^{\infty}(\Omega)^{2}\right)$ and $s \in L^{\infty}\left(\left[0, t_{0}\right) \times \Omega\right)$ with $s(t,.) \in W^{1, \infty}(\Omega)$. Let $y(t):=\left\|\left(u^{+}, u^{-}\right)(t, .)\right\|_{2}^{2}$. Then with Lemma 4.4 and Lemma 4.1, (20) it follows that

$$
\begin{aligned}
\frac{d}{d t} y(t) & \leq C_{2}\left(1+\left\|s_{0}\right\|_{W^{1, \infty}}+t^{1 / 4} y(t)^{1 / 2}\right) y(t) \\
& =a y(t)+b(t) y(t)^{3 / 2}
\end{aligned}
$$

with $a=C_{2}\left(1+\left\|s_{0}\right\|_{W^{1, \infty}}\right)$ and $b(t)=C_{2} t^{1 / 4}$. Let $z(t)$ denote the solution of the ODE

$$
\begin{aligned}
\dot{z}(t) & =a z(t)+b(t) z(t)^{3 / 2}, \\
z(0) & =y(0)=\left\|\left(u_{0}^{+}, u_{0}^{-}\right)\right\|_{2}^{2} .
\end{aligned}
$$

Then $0 \leq y(t) \leq z(t)$ as long as the solution $z(t)$ exists and $z(t)>0$ if $z(0)>0$. To solve (26) we set $w(t):=z(t)^{-1 / 2}$ and get

$$
\begin{aligned}
\dot{w}(t) & =-\frac{1}{2} a w(t)-\frac{1}{2} b(t) \\
w(0) & =z(0)^{-1 / 2} .
\end{aligned}
$$


Since the right hand side of (27) is strictly negative and bounded away from zero for all $t>0$ and $w_{0}>0$ the solution $w(t)$ will cross zero in finite time which leads to blow-up of $z$. We choose $0<\delta<w_{0}$ and $t^{*}=t^{*}\left(\delta, w_{0}\right)$ such that $w(t)>\delta$ for all $0 \leq t<t^{*}$ and $w\left(t^{*}\right)=\delta$. Then $t^{*}\left(\delta, w_{0}\right)$ is strictly increasing as a function of $w_{0}$ and decreasing as a function of $\delta$.

For variable initial conditions $s_{0}$ we have $t^{*}=t^{*}\left(w_{0}, \delta, a\right)$. So for given $C^{*} \geq\left\|u_{0}^{+}, u_{0}^{-}\right\|_{2}^{2}$, $\left(C^{*}=1 / \delta\right)$ we get

$$
\left\|\left(u^{+}, u^{-}\right)(t, .)\right\|_{2}^{2} \leq C^{*}
$$

for all solutions $\left(u^{+}, u^{-}\right) \in C^{1}\left([0, \theta), L^{\infty}(\Omega)\right)^{2}$, with $\theta=\min \left\{t_{0}, t^{*}\right\}$. By a density argument similar to this used in the proof of Lemma 4.2 this also holds for solutions $\left(u^{+}, u^{-}\right) \in L^{1}\left([0, \theta), L^{\infty}(\Omega)\right)^{2}$. The second estimate follows immediately from Lemma 4.1.

Notation: In the following we mark all constants which are related to the above a-priori estimate with "*".

\subsection{Proof of Theorem 3.1}

We use a contraction mapping principle twice. First we solve the $\left(u^{+}, u^{-}\right)$equations in (17) for arbitrary functions $s$ which satisfy the a-priori estimate of Theorem 4.5. Then we solve the whole system (17) for $u^{+}, u^{-}$and $s$. We use the following abbreviations for the Banach spaces: $X:=L^{\infty}\left(\left[0, t_{0}\right) \times \Omega\right)$ with

$$
\|u\|_{X}=\sup _{0 \leq t \leq t_{0}}\|u(t, .)\|_{\infty}
$$

and $Y:=L^{\infty}\left(\left[0, t_{0}\right), W^{1, \infty}(\Omega)\right)$ with

$$
\|s\|_{Y}=\sup _{0 \leq t \leq t_{0}}\left(\|s(t, .)\|_{\infty}+\left\|s_{x}(t, .)\right\|_{\infty}\right) .
$$

(i) Consider any $s \in Y$ with $\|s\|_{Y} \leq C^{* *}$ for all $0 \leq t \leq t_{0} \leq t^{*}$, where $C^{* *}$ and $t^{*}$ are given as in Theorem 4.5. Then from (A4) it follows that

$$
\mu^{ \pm}\left(s, s_{x}\right) \leq m\left(1+C^{* *}\right)=: m^{*} .
$$

(ii) Consider $\left(v^{+}, v^{-}\right) \in X^{2}$ with $v^{ \pm}(0,)=.u_{0}^{ \pm}$. We solve the initial value problem

$$
\begin{aligned}
u_{t}^{+}+\gamma u_{x}^{+} & =-\mu^{+}\left(s, s_{x}\right) v^{+}+\mu^{-}\left(s, s_{x}\right) v^{-} \\
u_{t}^{-}-\gamma u_{x}^{-} & =\mu^{+}\left(s, s_{x}\right) v^{+}-\mu^{-}\left(s, s_{x}\right) v^{-} \\
u^{ \pm}(0, .) & =u_{0}^{ \pm}
\end{aligned}
$$

on the whole line $\Omega=\mathbb{R}$ with the method of characteristics. In the case of Neumann boundary conditions it is shown in [23] that if $\mu^{ \pm}$satisfy the symmetry condition (A3) then the solution could be extended to a solution on the whole line in such a 
way that the restriction to $[-1,1]$ again satisfies Neumann boundary conditions. For $z_{0} \in \mathbb{R}$ let $z^{+}=z_{0}+\gamma t$ and $z^{-}=z_{0}-\gamma t$ denote the characteristics of (29). Then

$$
\begin{aligned}
& u^{+}\left(z^{+}\right)=u_{0}^{+}\left(z_{0}\right)+\int_{z_{0}}^{z^{+}}\left[-\left(\mu^{+} v^{+}\right)(y)+\left(\mu^{-} v^{-}\right)(y)\right] d y \\
& u^{+}\left(z^{-}\right)=u_{0}^{-}\left(z_{0}\right)+\int_{z_{0}}^{z^{-}}\left[-\left(\mu^{+} v^{+}\right)(y)+\left(\mu^{-} v^{-}\right)(y)\right] d y .
\end{aligned}
$$

The right hand sides define a solution operator $G=\left(G_{1}, G_{2}\right): X^{2} \rightarrow X^{2},\left(v^{+}, v^{-}\right) \mapsto$ $\left(u^{+}, u^{-}\right)$. To show that $G$ is a contraction for $t_{0}$ small enough we consider $\left(v^{+}, v^{-}\right),\left(w^{+}, w^{-}\right) \in X^{2}$ with $v^{ \pm}(0,)=.w^{ \pm}(0,)=.u_{0}^{ \pm}$. Then

$$
\begin{aligned}
\left\|G_{1}\left(\begin{array}{c}
v^{+} \\
v^{-}
\end{array}\right)-G_{1}\left(\begin{array}{c}
w^{+} \\
w^{-}
\end{array}\right)\right\|_{X} & =\left\|\int_{z_{0}}^{z^{+}}\left(\mu^{+}\left(w^{+}-v^{+}\right)+\mu^{-}\left(v^{-}-w^{-}\right)\right) d y\right\|_{X} \\
& \leq \gamma t_{0} m^{*}\left(\left\|v^{+}-w^{+}\right\|_{X}+\left\|v^{-}-w^{-}\right\|_{X}\right) \\
& \leq 2 \gamma t_{0} m^{*} \max \left\{\left\|v^{+}-w^{+}\right\|_{X},\left\|v^{-}-w^{-}\right\|_{X}\right\} .
\end{aligned}
$$

A similar estimate holds for $G_{2}$. Hence for

$$
t_{0}<\frac{1}{2 \gamma m^{*}}
$$

$G$ is a contraction. So $G$ has a unique fixed point $\left(u^{+}, u^{-}\right) \in X^{2}$, which depends on $s$. By (30), (31) and (32) it follows that

$$
\left\|u^{+}\right\|_{X}+\left\|u^{-}\right\|_{X} \leq\left\|u_{0}^{+}\right\|_{\infty}+\left\|u_{0}^{-}\right\|_{\infty}+2 \gamma t_{0} m^{*}\left(\left\|u^{+}\right\|_{X}+\left\|u^{-}\right\|_{X}\right) .
$$

So

$$
\left\|u^{+}\right\|_{X}+\left\|u^{-}\right\|_{X} \leq \frac{1}{1-2 \gamma m^{*} t_{0}}\left(\left\|u_{0}^{+}\right\|_{\infty}+\left\|u_{0}^{-}\right\|_{\infty}\right) .
$$

This also holds in case of Neumann boundary conditions.

(iii) Now it remains to solve the full system for $s$. Consider $\tilde{s} \in Y$ and let $\left(\tilde{u}^{+}, \tilde{u}^{-}\right) \in X^{2}$ denote the corresponding fixed point of $G$ in (ii). Let $s$ denote the solution of the initial value problem

$$
\begin{aligned}
s_{t} & =D s_{x x}-\beta s+\alpha\left(\tilde{u}^{+}+\tilde{u}^{-}\right) \\
s(0, .) & =s_{0}
\end{aligned}
$$

Then

$$
s(t, x)=T(t) s_{0}(x)+\alpha \int_{0}^{t} T(t-\theta)\left(\tilde{u}^{+}+\tilde{u}^{-}\right)(\theta, x) d \theta
$$

where $T(t)$ fulfills the properties mentioned in connection with (19). Because of Lemma 4.1, (21) the right hand side of (36) defines a solution operator $Q: Y \rightarrow Y$. For suitable $M>0$ large enough and $t_{0}>0$ small enough we will show that

$$
Q: B_{Y}(M, 0) \rightarrow B_{Y}(M, 0)
$$


where $B_{Y}(M, 0)$ denotes the $M$-ball in $Y$ and $Q$ is a contraction on $B_{Y}(M, 0)$. Let $C^{*}, C^{* *}>0$ denote the constants in Theorem 4.5. For given $\varepsilon>0$ we choose $C^{*}$ large enough such that

$$
C^{* *}>2 C\left(\left\|s_{0}\right\|_{W^{1, \infty}}+\varepsilon\right)
$$

and $0<t_{0}<1 /\left(2 \gamma m^{*}\right)$ small enough such that

$$
\frac{t_{0}^{1 / 2}}{1-\gamma m^{*} t_{0}}\left(\left\|u_{0}^{+}\right\|_{\infty}+\left\|u_{0}^{-}\right\|_{\infty}\right)<\varepsilon
$$

Then for $s \in B_{Y}\left(C^{* *}, 0\right)$ with (21) and (34) it follows that

$$
\begin{aligned}
\|Q(s)\|_{Y} & \leq 2 C_{\infty}\left(\left\|s_{0}\right\|_{W^{1, \infty}}+t_{0}^{1 / 2}\left\|u^{+}+u^{-}\right\|_{X}\right) \\
& \leq 2 C_{\infty}\left(\left\|s_{0}\right\|_{W^{1, \infty}}+\frac{t_{0}^{1 / 2}}{1-\gamma m^{*} t_{0}}\left(\left\|u_{0}^{+}\right\|_{\infty}+\left\|u_{0}^{-}\right\|_{\infty}\right)\right) \\
& <C^{* *}
\end{aligned}
$$

Hence with $M=C^{* *}$ the operator $Q$ satisfies (37). To show that $Q$ is a contraction in $B_{Y}\left(C^{* *}, 0\right)$ we consider $s_{1}, s_{2} \in B_{Y}\left(C^{* *}, 0\right)$ with $s_{1}(0,)=.s_{2}(0,)=.s_{0}$. Let $\left(u_{1}^{+}, u_{1}^{-}\right)$and $\left(u_{2}^{+}, u_{2}^{-}\right)$denote the corresponding fixed points of $G$. Then with (21) it follows that

$$
\begin{aligned}
\left\|Q\left(s_{1}\right)-Q\left(s_{2}\right)\right\|_{Y} & =\left\|\alpha \int_{0}^{t} T(t-\theta)\left[u_{1}^{+}+u_{1}^{-}-u_{2}^{+}-u_{2}^{-}\right] d \theta\right\|_{Y} \\
& \leq C_{\infty} t_{0}^{1 / 2}\left(\left\|u_{1}^{+}-u_{2}^{+}\right\|_{X}+\left\|u_{1}^{-}-u_{2}^{-}\right\|_{X}\right)
\end{aligned}
$$

Now we represent $\left\|u_{1}^{+}-u_{2}^{+}\right\|_{X}$ and $\left\|u_{1}^{-}-u_{2}^{-}\right\|_{X}$ by using (30) with $u_{1}^{ \pm}$and $u_{2}^{ \pm}$instead of $v^{ \pm}$, respectively. Set $\mu_{i}^{ \pm}:=\mu^{ \pm}\left(s_{i}, s_{i, x}\right)$ for $i=1,2$ then

$$
\begin{aligned}
&\left\|u_{1}^{+}-u_{2}^{+}\right\|_{X}=\left\|\int_{z_{0}}^{z^{+}}\left(-\mu_{1}^{+} u_{1}^{+}+\mu_{1}^{-} u_{1}^{-}+\mu_{2}^{+} u_{2}^{+}-\mu_{2}^{-} u_{2}^{-}\right) d z\right\|_{X} \\
&= \| \int_{z_{0}}^{z^{+}}\left(-\mu_{1}^{+}\left(u_{1}^{+}-u_{2}^{+}\right)-\left(\mu_{1}^{+}-\mu_{2}^{+}\right) u_{2}^{+}\right. \\
&\left.\quad+\mu_{1}^{-}\left(u_{1}^{-}-u_{2}^{-}\right)+\left(\mu_{1}^{-}-\mu_{2}^{-}\right) u_{2}^{-}\right) d z \|_{X} \\
& \leq \quad \gamma t_{0} m^{*}\left(\left\|u_{1}^{+}-u_{2}^{+}\right\|_{X}+\left\|u_{2}^{+}\right\|_{X} l\left\|s_{1}-s_{2}\right\|_{Y}\right. \\
&\left.\quad+\left\|u_{1}^{-}-u_{2}^{-}\right\|_{X}+\left\|u_{2}^{-}\right\|_{X} l\left\|s_{1}-s_{2}\right\|_{Y}\right),
\end{aligned}
$$

where $l$ is the Lipschitz constant of $\mu^{ \pm}$(due to (A4)). A similar estimate holds for $\left\|u_{1}^{-}-u_{2}^{-}\right\|_{X}$. Hence for $t_{0}<1 /\left(2 \gamma m^{*}\right)$ we observe

$$
\left\|u_{1}^{+}-u_{2}^{+}\right\|_{X}+\left\|u_{1}^{-}-u_{2}^{-}\right\|_{X} \leq \frac{2 l \gamma t_{0}}{1-2 \gamma m^{*} t_{0}}\left(\left\|u_{2}^{+}\right\|_{X}+\left\|u_{2}^{-}\right\|_{X}\right)\left\|s_{1}-s_{2}\right\|_{Y} .
$$


So using (34) we get

$$
\left\|Q\left(s_{1}\right)-Q\left(s_{2}\right)\right\|_{Y} \leq C_{\infty} t_{0}^{1 / 2} \frac{2 l \gamma t_{0}}{\left(1-2 \gamma m^{*} t_{0}\right)^{2}}\left(\left\|u_{0}^{+}\right\|_{\infty}+\left\|u_{0}^{-}\right\|_{\infty}\right)\left\|s_{1}-s_{2}\right\|_{Y} .
$$

Now we choose $t_{0}<\min \left(t^{*}, 1 /\left(2 \gamma m^{*}\right)\right)$ small enough such that

$$
\frac{2 C_{\infty} l \gamma t_{0}^{3 / 2}}{\left(1-2 \gamma m^{*} t_{0}\right)^{2}}\left(\left\|u_{0}^{+}\right\|_{\infty}+\left\|u_{0}^{-}\right\|_{\infty}\right)<1
$$

Then the solution $s$ is the unique fixed point of $Q: B_{Y}\left(C^{* *}, 0\right) \rightarrow B_{Y}\left(C^{* *}, 0\right)$.

(iv) Assume that $t_{0}$ is the maximal time of existence and for $t \leq t_{0}$ we have $\|s\|_{Y} \leq$ $K<\infty$. Then with (A4) we get an estimate of the form (28) and with arguments like before the solution can be continued to some $t_{0}+\varepsilon$. Hence the maximal time of existence $T_{\max }$ is specified by

$$
\lim _{t \rightarrow T_{\max }}\|s(t, .)\|_{W^{1, \infty}}=\infty .
$$

\subsection{Proof of Corollary 3.2}

Here we replace (A4) by (A4'). Local existence of solutions is clear due to Theorem 3.1. For global existence we recall the a-priori estimates of section 3.1 and derive a global estimate for $s$ in $W^{1, \infty}$. Lemma 4.1, Lemma 4.2 and Corollary 4.3 hold without modification. In the proof of Lemma 4.4 we change (25) into

$$
\|\xi(t, .)\|_{\infty} \leq m^{*}
$$

where $m^{*}$ is given by (A4'). Then the following global estimates for $\left(u^{+}, u^{-}\right)$in $L^{2}$ and for $s$ in $W^{1, \infty}$ hold:

Lemma 4.6 Assume (A1)-(A3) and (A4'). Then for each $t \geq 0$ we have

$$
\begin{aligned}
\left\|\left(u^{+}, u^{-}\right)(t, .)\right\|_{2}^{2} & \leq\left\|\left(u_{0}^{+}, u_{0}^{-}\right)\right\|_{2}^{2} e^{2 m^{*} t} \\
\|s(t, .)\|_{W^{1, \infty}} & \leq C_{2}\left(\left\|s_{0}\right\|_{W^{1, \infty}}+2 t^{1 / 2}\left\|\left(u_{0}^{+}, u_{0}^{-}\right)\right\|_{2} e^{m^{*} t}\right) .
\end{aligned}
$$

From Theorem 3.1 it follows that the maximal time of existence of solutions is $T_{\max }=\infty$.

\section{The case of $\tau=0$}

\section{$5.1 \quad$ A-priori estimates}

For $\tau=0$ system (17) reduces to (18). Let $u^{ \pm} \in L^{1}\left(\left[0, t_{0}\right) \times \Omega\right)$ be given and

$$
s_{x x}=\beta s-\alpha\left(u^{+}+u^{-}\right) .
$$


In case of initial conditions (bc1), $s$ satisfies homogeneous Dirichlet boundary conditions on the convex hull of the union of the support of $u^{+}(t,$.$) and u^{-}(t,$.$) for each time$ $0 \leq t \leq t_{0}$.

The a-priori estimates and the local existence results of the previous chapter do not apply. So we have to mimic the essential steps. First the positivity of $s$ follows from the elliptic maximum principle.

Lemma 5.1 Assume $u^{+} \geq 0$ and $u^{-} \geq 0$ then $s(t, x) \geq 0$ for all $(t, x) \in\left[0, t_{0}\right) \times \mathbb{R}$.

As in the previous section $u^{ \pm}$stay positive:

Lemma 5.2 The solution satisfy $u^{ \pm}(t, x) \geq 0$ as long as they exist. Hence

$$
u(t, x) \geq|v(t, x)|
$$

The proof follows the lines of the proof of Lemma 4.2.

Next we state the global $W^{1, \infty}$ estimate for $s$ :

Lemma 5.3 Consider $u^{+}(t,),. u^{-}(t,.) \in L^{1}(\Omega)$ for $0 \leq t<\infty$ and let $R(t) \leq 1$ such that $\operatorname{supp}(s(t,).) \subset(-R(t), R(t))$ for $(\mathrm{bc} 1)$ and $R(t)=1$ for $(\mathrm{bc} 2)$. Then

$$
\begin{aligned}
& \|s(t, .)\|_{1}=\frac{\alpha}{\beta}\left\|\left(u^{+}+u^{-}\right)(t, .)\right\|_{1} \\
& \left\|s_{x}(t, .)\right\|_{\infty} \leq \alpha\left\|\left(u^{+}+u^{-}\right)(t, .)\right\|_{1} \\
& 0 \leq s(t, x) \leq \phi(t)\left\|\left(u^{+}+u^{-}\right)(t, .)\right\|_{1},
\end{aligned}
$$

where

$$
\phi(t)=\frac{\alpha}{2 R(t)}\left(\frac{1}{\beta}+4 R^{2}(t)\right) .
$$

Furthermore we can choose $R(t)=x_{0}+\gamma t$ for some $x_{0}>0$ large enough such that $\phi(t)$ is increasing and bounded for all $t \geq 0$.

Proof:

(i) Since

$$
\int_{\Omega} s_{x x} d x=\beta \int_{\Omega} s d x-\alpha \int_{\Omega}\left(u^{+}+u^{-}\right) d x
$$

and $s \geq 0$ (Lemma 5.1), we end up for (bc1) and (bc2) with equation (i).

(ii) We integrate the $s$ equation along $[-R(t), x]$ so we have

$$
s_{x}(t, x)=s_{x}(t,-R(t))+\beta \int_{-R(t)}^{x} s d y-\alpha \int_{-R(t)}^{x}\left(u^{+}+u^{-}\right) d y .
$$

Since $s_{x}(t,-R(t))=0$ and $u \geq 0$ (Lemma 5.2) it follows that $\left\|s_{x}(t, .)\right\|_{\infty} \leq \beta\|s(t, .)\|_{1}$ Estimate $(i i)$ follows from $(i)$. 
(iii) Here we follow the ansatz used by Nagai [36]. Consider

$$
\begin{aligned}
\int_{-R(t)}^{R(t)} \int_{y}^{x} s_{z}(t, z) d z d y & =\int_{-R(t)}^{R(t)}(s(t, x)-s(t, y)) d y \\
& =2 R(t) s(t, x)-\int_{-R(t)}^{R(t)} s(t, y) d y
\end{aligned}
$$

Using $(i)$ and $(i i)$ it follows that

$$
\begin{aligned}
s(t, x) & =\frac{1}{2 R(t)}\left(\int_{-R(t)}^{R(t)} s(t, y) d y+\int_{-R(t)}^{R(t)} \int_{y}^{x} s_{z}(t, z) d z d y\right) \\
& \leq \frac{\alpha}{2 R(t)}\left(\frac{1}{\beta}+(2 R(t))^{2}\right)\left\|\left(u^{+}+u^{-}\right)(t, .)\right\|_{1}
\end{aligned}
$$

which is $(i i i)$.

Finally we study the growth properties of $\phi(t)$. For (bc2) we have $R(t)=1$ and $\phi(t)=$ $\frac{\alpha}{2}\left(\frac{1}{\beta}+4\right)$. For $(\mathrm{bc} 1)$ we define

$$
\begin{aligned}
x_{\min } & :=\min \left\{\operatorname{supp}\left(u_{0}^{+}\right) \cup \operatorname{supp}\left(u_{0}^{-}\right)\right\}-1 \\
x_{\max } & :=\max \left\{\operatorname{supp}\left(u_{0}^{+}\right) \cup \operatorname{supp}\left(u_{0}^{-}\right)\right\}+1 .
\end{aligned}
$$

and we set $x_{0}:=\max \left\{-x_{\min }, x_{\max }\right\}$. Then for $t \geq 0$ we choose $R(t)=x_{0}+\gamma t$. If necessary we increase $x_{0}$ above $\sqrt{1 /(4 \beta)}$ such that $\phi^{\prime}(t) \geq 0$ for all $t \geq 0$.

\subsection{Proof of Theorem 3.3}

For $\tau=0$ local existence will be proven by a contraction mapping principle like in Theorem 3.1. Global existence follows from the global estimates in Lemma 5.3. Again for $t_{0}>0$ small enough we use $X=L^{\infty}\left(\left[0, t_{0}\right) \times \Omega\right)$ and $Y=L^{\infty}\left(\left[0, t_{0}\right), W^{1, \infty}(\Omega)\right)$. Let $M_{1}>0$ be such that $\left\|u_{0}^{+}\right\|_{\infty}+\left\|u_{0}^{-}\right\|_{\infty} \leq M_{1}$. Because of the assumptions on the initial data there exists a constant $c_{1}>0$ such that

$$
\left\|u_{0}^{+}\right\|_{1}+\left\|u_{0}^{-}\right\|_{1} \leq c_{1} M_{1} .
$$

(i) We define $R(t)$ and $\phi(t)$ as in Lemma 5.3 and consider $s \in Y$ with

$$
\|s\|_{Y} \leq\left(\alpha+\phi\left(t_{0}\right)\right) c_{1} M_{1} .
$$

Then from (A4) it follows that

$$
\mu^{ \pm}\left(s, s_{x}\right) \leq m\left(1+\left(\alpha+\phi\left(t_{0}\right) c_{1} M_{1}\right)=: m^{*}\left(t_{0}\right) .\right.
$$

(ii) To solve

$$
\begin{aligned}
u_{t}^{+}+\gamma u_{x}^{+} & =-\mu^{+}\left(s, s_{x}\right) u^{+}+\mu^{-}\left(s, s_{x}\right) u^{-} \\
u_{t}^{-}-\gamma u_{x}^{-} & =\mu^{+}\left(s, s_{x}\right) u^{+}-\mu^{-}\left(s, s_{x}\right) u^{-} \\
u^{ \pm}(0, .) & =u_{0}^{ \pm}
\end{aligned}
$$


for a given $s \in Y$ we follow part (ii) of the proof of Theorem 3.1. Here $m^{*}=m^{*}\left(t_{0}\right)$ and hence condition (33) changes into

$$
t_{0}<\frac{1}{2 \gamma m^{*}\left(t_{0}\right)}
$$

Since $m^{*}\left(t_{0}\right)$ is increasing in $t_{0}$ and $m^{*}(0)>0$, there exists a nonempty interval $[0, \bar{t}]$ such that (41) is satisfied for all $0 \leq t_{0} \leq \bar{t}$. Let $t_{0}>0$ be fixed then

$$
\left\|u^{+}\right\|_{X}+\left\|u^{-}\right\|_{X} \leq \frac{1}{1-2 \gamma m^{*}\left(t_{0}\right) t_{0}}\left(\left\|u_{0}^{+}\right\|_{\infty}+\left\|u_{0}^{-}\right\|_{\infty}\right) .
$$

Equation (40) conserves total mass, hence

$$
\left\|\left(u^{+}+u^{-}\right)(t, .)\right\|_{1}=\left\|\left(u_{0}^{+}, u_{0}^{-}\right)\right\|_{1} \leq c_{1} M_{1}, \quad \forall 0 \leq t \leq t_{0} .
$$

(iii) To solve system (18) we again use a fixed point argument. Let $\rho:=\left(\alpha+\phi\left(t_{0}\right)\right) c_{1} M_{1}$ and let $\tilde{s} \in Y$ satisfy $\|\tilde{s}\|_{Y} \leq \rho$. With $\left(\tilde{u}^{+}, \tilde{u}^{-}\right)$we denote the corresponding solution of $(40)$. If $s(t, x)$ is the solution of

$$
s_{x x}=\beta s-\alpha\left(\tilde{u}^{+}+\tilde{u}^{-}\right)
$$

for each $0 \leq t \leq t_{0}$ it follows with Lemma 5.3 that

$$
\begin{aligned}
\|s\|_{Y} & \leq\left(\alpha+\phi\left(t_{0}\right)\right)\left\|\left(\tilde{u}^{+}+\tilde{u}^{-}\right)(t, .)\right\|_{1} \\
& \leq\left(\alpha+\phi\left(t_{0}\right)\right) c_{1} M_{1}=\rho .
\end{aligned}
$$

If $Q: Y \rightarrow Y$ denotes the solution operator which maps $\tilde{s}$ onto $s$ it follows that $Q: B_{Y}(\rho, 0) \rightarrow B_{Y}(\rho, 0)$. It remains to show that $Q$ is a contraction. Consider $s_{1}, s_{2} \in B_{Y}(\rho, 0)$ and let $\left(u_{1}^{+}, u_{1}^{-}\right),\left(u_{2}^{+}, u_{2}^{-}\right)$denote the corresponding solutions of (40). Then the difference $\sigma:=s_{1}-s_{2}$ satisfies

$$
\sigma_{x x}=\beta \sigma-\alpha\left(u_{1}^{+}-u_{2}^{+}+u_{1}^{-}-u_{2}^{-}\right) .
$$

Lemma 5.3 yields

$$
\|\sigma\|_{Y} \leq\left(\alpha+\phi\left(t_{0}\right)\right) c_{1}\left(\left\|u_{1}^{+}-u_{2}^{+}\right\|_{X}+\left\|u_{1}^{-}-u_{2}^{-}\right\|_{X}\right) .
$$

In order to estimate $\left\|u_{1}^{+}-u_{2}^{+}\right\|_{X}+\left\|u_{1}^{-}-u_{2}^{-}\right\|_{X}$ we recall estimate (39) in the proof of Theorem 3.1 and get

$$
\left\|u_{1}^{+}-u_{2}^{+}\right\|_{X}+\left\|u_{1}^{-}-u_{2}^{-}\right\|_{X} \leq \frac{2 l \gamma t_{0}}{1-2 \gamma m^{*}\left(t_{0}\right) t_{0}}\left(\left\|u_{2}^{+}\right\|_{X}+\left\|u_{2}^{-}\right\|_{X}\right)\left\|s_{1}-s_{2}\right\|_{Y} .
$$

We estimate $\left\|u_{2}^{+}\right\|_{X}+\left\|u_{2}^{-}\right\|_{X}$ by (42) and choose $t_{0}<1 /\left(2 \gamma m^{*}\left(t_{0}\right)\right)$ small enough, such that

$$
\frac{2 l \gamma c_{1}\left(\alpha+\phi\left(t_{0}\right)\right) t_{0}}{\left(1-2 \gamma m^{*}\left(t_{0}\right) t_{0}\right)^{2}}\left(\left\|u_{0}^{+}\right\|_{\infty}+\left\|u_{0}^{-}\right\|_{\infty}\right)<1 .
$$

Then the fixed point argument gives the desired result. 
(iv) If $\left\|s\left(t_{0}, .\right)\right\|_{W^{1, \infty}}<\infty$ for some $t_{0}>0$ we can continue the solution up to some $t_{0}+\varepsilon$ by using arguments of the above proof. Since system (40) preserves total mass it follows from Lemma 5.3 that $\|s(t, .)\|_{W^{1, \infty}}<\infty$ for all $t \geq 0$. Hence the solution exists globally.

\section{Stationary solutions and numerical examples}

To give an impression on the rich qualitative behavior of system (1) we show some numerical examples and compare the resulting patterns to the ones in the related parabolic models. First we investigate stationary solutions of (1), which are candidates for globally existing patterns.

$$
\begin{aligned}
\gamma \bar{u}_{x}^{+} & =-\mu^{+} \bar{u}^{+}+\mu^{-} \bar{u}^{-}, \\
\gamma \bar{u}_{x}^{-} & =-\mu^{+} \bar{u}^{+}+\mu^{-} \bar{u}^{-}, \\
D \bar{s}_{x x} & =\beta \bar{s}-\alpha\left(\bar{u}^{+}+\bar{u}^{-}\right) .
\end{aligned}
$$

So $\bar{v}_{x}=\bar{u}_{x}^{+}-\bar{u}_{x}^{-}=0$, hence $\bar{v}(x) \equiv$ const. For both cases of boundary conditions, (bc1) and (bc2), it follows that $\bar{v}=0$. Define $w:=\bar{u}^{+}=\bar{u}^{-}$, then

$$
w_{x}=\frac{1}{\gamma}\left(\mu^{-}-\mu^{+}\right) w
$$

For the numerical simulations we choose parameters which compare to those of the parabolic chemotaxis system given by Othmer and Stevens [39], to approximate the reinforced random walk given by Davis [10]:

$$
\begin{aligned}
u_{t} & =A\left(u_{x}-\chi(s) u s_{x}\right)_{x} \\
s_{t} & =f(u, x) \\
A & :=0.036 \\
\chi(s) & :=\frac{\kappa}{s} \\
\mu^{ \pm}(s) & :=\left(\frac{\gamma^{2}}{2 A} \mp \chi(s) s_{x}\right)^{+},
\end{aligned}
$$

$\kappa$ and $\gamma$ will be specified later. The turning rates are chosen such that the parabolic limit can formally be observed. When $D=0$, the equation for stationary solutions (45) with the above choice of parameters reads

$$
w_{x}=\frac{1}{\gamma} \frac{1}{1+2 \alpha / \beta w} w_{x} w
$$

which is solved in $L^{\infty}$ only if $w_{x}=0$ a.e.. This means, that for model (17) with parameters chosen as in (47) there exists no global stationary pattern. If we chose additionally $\beta=0$, then $w=0$. Nevertheless aggregation can clearly be observed. In the following examples 
we choose Neumann boundary conditions (bc2) on the interval $[0,1]$. For the time evolution we take a Godunov-Type method of first order (see e.g. LeVeque [32]). Initial conditions for Fig.1 and 2 are

$$
\begin{aligned}
u_{0}^{ \pm}(x) & =0.5+0.005 \cos (2 \pi x) \\
s_{0} & =1
\end{aligned}
$$

In Fig. 1 the time evolution of the total density $u$ is shown up to time $t=500$ with $\gamma=0.5$ and $\kappa=1$.

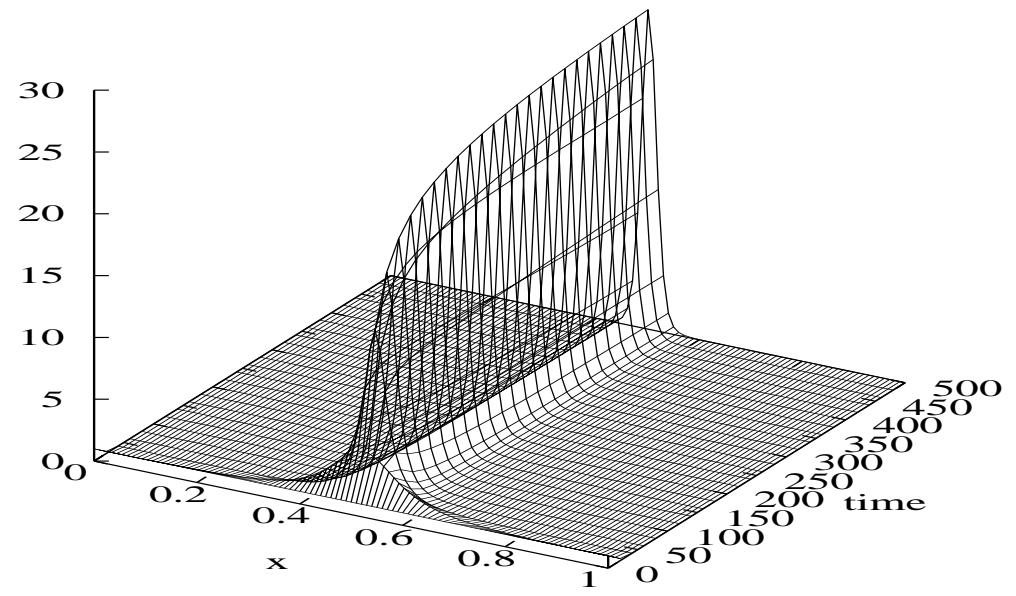

Fig.1: $\gamma=0.5, d x=d t=0.01, \kappa=1$

Here one clearly observes the aggregation effect. Although this localized solution does not approach a stationary pattern, we do not expect finite time blow up. To prove this, a more detailed analysis is required.

In the next example we consider nonlinear reproduction of the external stimulus: $f\left(s, u^{+}+\right.$ $\left.u^{-}\right)=s\left(u^{+}+u^{-}\right)$, which has been called exponential growth in [39]. This case is not covered by our existence results above. The time evolution of the total density $u$, which is shown in Fig.2 strongly indicates finite time blow up. The parameter values in this example compare to those presented in [39] for the parabolic model, with the exception that we choose $\kappa=0.1$ to observe blow up near $T=10$. Still the parabolic model blows up in finite time also for $\kappa=0.1$. For $\kappa=1$ Othmer and Stevens observed blow up at $T=9.3$. In this case the numerics for the hyperbolic system are roughly unstable. As expected, the dynamics of the hyperbolic model seem to be more unstable than those of the parabolic model.

The regularization of the analytical semigroup of the parabolic problem stabilizes the system compared to a model with finite range of dependence. 


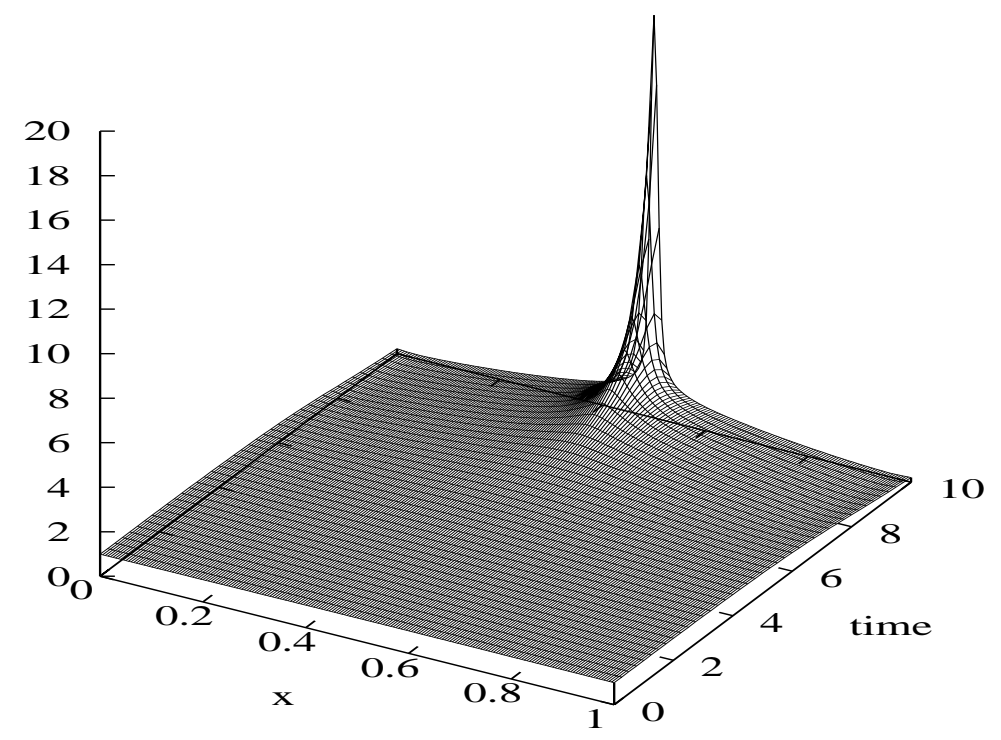

Fig.2: $\gamma=0.1, d x=0.01, d t=0.05, \kappa=0.1$

The last example shows the dynamics of a swarm which initially is located at the subinterval $[0.2,0.6]$. Here again we consider linear growth of the external signal, but the chemotactic sensitivity is $\chi(s)=\kappa /(1+s)$. The initial distribution of the external stimulus is increasing along the swarm and has a maximum at $x=0.8$. We observe that the production of the external signal is strong enough to cause two aggregation centers (Fig.3).

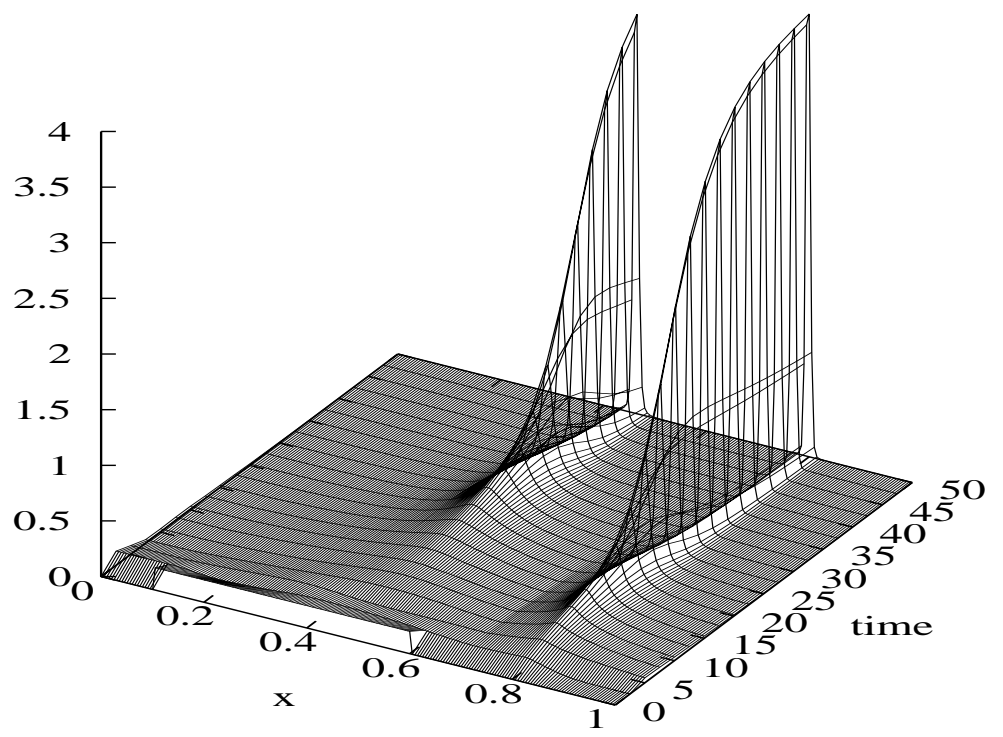

Fig.3: $\gamma=0.1, d x=0.005, d t=0.0025, \kappa=1$ 
Forthcoming studies and a detailed analysis have to clarify the asymptotic behavior, the patterns one can expect and finite time blowup for the hyperbolic chemotaxis model we proposed in (1).

\section{References}

[1] W. Alt. Biased random walk model for chemotaxis and related diffusion approximation. J. Math. Biology, 9:147-177, 1980.

[2] W. Alt. Singular perturbation of differential integral equations describing biased random walks. J. für die reine und angewandte Mathematik, 322:15-41, 1981.

[3] H.C. Berg and D.A. Brown. Chemotaxis in Escherichia coli analyzed by threedimensional tracing. Antibiotics and Chemoterapy, 19:55-78, 1974.

[4] P. Biler. Growth and accretion of mass in an astrophysical model. Appl. Math., 23(2):179-189, 1995.

[5] P. Biler and T. Nadzieja. Growth and accretion of mass in an astrophysical model II. Appl. Math., 23(3):351-361, 1995.

[6] K.C. Chen, R.M. Ford, and P.T. Cummings. Mathematical models for motile bacterial transport in cylindrical tubes. Preprint, 1998.

[7] K.C. Chen, R.M. Ford, and P.T. Cummings. Perturbation expansion of Alt's cell balance equations reduces to Segel's $1 \mathrm{~d}$ equation for shallow chemoattractant gradients. SIAM J. Appl. Math., 1998. in press.

[8] S. Childress. Chemotactic collapse in two dimensions. Lect. Notes in Biomath., 55:61-68, 1984.

[9] S. Childress and J.K. Percus. Nonlinear aspects of chemotaxis. Math. Biosci., 56:217$237,1981$.

[10] B Davis. Reinforced random walks. Prob. Thy. Rel. Fields, pages 203-229, 1990.

[11] R.M. Ford and P.T. Cummings. On the relationship between cell balance equations for chemotactic cell populations. SIAM Appl. Math., 52:1426-141, 1992.

[12] R.M. Ford and P.T. Cummings. Errata to: On the relationship between cell balance equations for chemotactic cell populations. SIAM J. Appl. Math., 53:1505, 1993.

[13] H. Gajewski and K. Zacharias. Global behavior of a reaction-diffusion system modelling chemotaxis. WIAS Preprint, (232), 1996.

[14] J. I. Gallin and P. G. Quie. Leukocyte chemotaxis: methods, physiology, and clinical implications. Raven Press, New York, 1978. 
[15] S. Goldstein. On diffusion by discontinuous movements and the telegraph equation. Quart. J. Mech. Appl. Math., 4:129-156, 1951.

[16] K.P. Hadeler. Travelling fronts for correlated random walks. Canadian Applied Math. Quart., 2, 1994.

[17] K.P. Hadeler. Random walk systems and reaction telegraph equations. In S. v. Strien and S.V. Lunel, editors, Dynamical Systems and their Applications in Science. Royal Academy of the Netherlands, 1995.

[18] K.P. Hadeler. Travelling fronts in random walk systems. FORMA, Japan, 10(3):223$233,1995$.

[19] K.P. Hadeler. Spatial epidemic spread with slow infectives. In R.J. Jarvis, editor, Ordinary and Partial Differential Equations, 1996.

[20] M. Herrero and J. Velázquez. Chemotactic collapse for the Keller-Segel model. J. Math. Biol., 35:177-194, 1996.

[21] M. Herrero and J. Velázquez. Singularity patterns in a chemotaxis model. Math. Annalen, 306:583-623, 1996.

[22] T. Hillen. Nichtlineare hyperbolische Systeme zur Modellierung von Ausbreitungsvorgängen und Anwendung auf das Turing Modell. Dissertation, Uni. Tübingen, 1995.

[23] T. Hillen. Nonlinear hyperbolic systems describing random motion and their application on the Turing model. Dissertation Summaries in Math., 1:121-128, 1996.

[24] T. Hillen. Qualitative analysis of hyperbolic random walk systems. SFB 382, Report Nr:, 43, 1996.

[25] T. Hillen. A Turing model with correlated random walk. J. Math. Biol., 35:49-72, 1996.

[26] T. Hillen. Invariance principles for hyperbolic random walk systems. J. Math. Ana. Appl., 210:360-374, 1997.

[27] T. Hillen and C. Rohde. Viscosity solutions of hyperbolic models for chemotaxis. 1998. In preparation.

[28] E.E. Holmes. Are diffusion models too simple? A comparison with telegraph models of invasion. American Naturalist, 142:779-795, 1993.

[29] W. Jäger and S. Luckhaus. On explosions of solutions to a system of partial differential equations modelling chemotaxis. Trans. AMS, 329:819-824, 1992.

[30] M. Kac. A stochastic model related to the telegrapher's equation. Rocky Mountain J. Math., 4:497-509, 1974. 
[31] E.F. Keller and L.A. Segel. Initiation of slime mold aggregation viewed as an instability. J. Theo. Biology, 26:399-415, 1970.

[32] R.J. LeVeque. Numerical Methods for Conseravtion Laws. Birkhäuser, Basel, 1992.

[33] H.A. Levine and B.D. Sleeman. A system of reaction diffusion equations arising in the theory of reinforced random walks. SIAM J. Appl. Math., 57:683-730, 1997.

[34] J. Müller and T. Hillen. Modulation equations and parabolic limits of reaction random walk systems. Math. Methods in Appl. Sci., 21:1207-1226, 1998.

[35] J.D. Murray. Mathematical Biology. Springer, 1989.

[36] T. Nagai. Blow up of radially symmetric solutions to a chemotaxis system. Adv. Math. Sci. Appl., 5:581-601, 1995.

[37] H.G. Othmer, S.R. Dunbar, and W. Alt. Models of dispersal in biological systems. J. Math. Biol., 26:263-298, 1988.

[38] H.G. Othmer and P. Schaap. Oscillatory cAMP signaling in the development of dictyostelium discoideum. Comments on Theor. Biology, 1998. to appear.

[39] H.G. Othmer and A. Stevens. Aggregation, blowup and collapse: The ABC's of taxis in reinforced random walks. SIAM J. Appl. Math., 57:1044-1081, 1997.

[40] A. Pazy. Semigroups of Linear Operators and Applications to Partial Differential Equations. Springer, New York, 1983.

[41] B. Pfistner. A one dimensional model for the swarming behavior of Myxobakteria. In W. Alt and G. Hoffmann, editors, Biological Motion. Lect. Notes on Biomath., 89, Springer, 1990.

[42] M.A. Rivero, R.T. Tranquillo, H.M. Buettner, and D.A. Lauffenburger. Transport models for chemotactic cell populations based on individual cell behavior. Chem. Eng. Sciences, 44:1-17, 1989.

[43] L.A. Segel. A theoretical study of receptor mechanisms in bacterial chemotaxis. SIAM Appl. Math., 32:653-665, 1977.

[44] T. Senba. Blow-up of radially symmetric solutions to some systems of partial differential equations modelling chemotaxis. Adv. Math. Sci. Appl., 7:79-92, 1997.

[45] D.R. Soll. Behavioral studies into the mechanism of eukaryotic chemotaxis. J. Chemical Ecology, 16:133-150, 1990.

[46] A. Stevens. A model for gliding and aggregation of myxobacteria. In M. Markus A. Holden and H. G. Othmer, editors, Proc. Conf. on Nonlinear Wave Processes in Excitable Media, pages 269-276. Plenum Press, New York, 1991. 
[47] A. Stevens. Derivation of chemotaxis-equations as limit dynamics of moderately interacting stochastic many particle systems. 1998. submitted.

[48] A. Stevens. A stochastic cellular automaton, modeling gliding and aggregation of myxobacteria. 1998. submitted.

[49] D.W. Stroock. Some stochastic processes which arise from a model of the motion of a bacterium. Probab. Theory and Related Fields, 28:305-315, 1974.

[50] M.E. Taylor. Partial Differential Equations III. Springer, New York, 1996. 